\title{
Electrostatic Solitary Waves in Pair-ion Plasmas with Trapped Electrons
}

\author{
A. Mushtaq • M. Ikram • R. E. H. Clark
}

Received: 29 June 2014 / Published online: 23 September 2014

(c) Sociedade Brasileira de Física 2014

\begin{abstract}
Electrostatic solitons in an unmagnetized pair-ion plasma comprising adiabatic fluid positive and negative ions and non-isothermal electrons are investigated using both arbitrary and small amplitude techniques. An energy integral equation involving the Sagdeev potential is derived, and the basic properties of large amplitude solitary structures are investigated. Various features of solitons differ in different existence domains. The effects of ion adiabaticity, particle concentration, and resonant electrons on the profiles of Sagdeev potential and corresponding solitary waves are investigated. The generalized Korteweg-de Vries equation with mixed-nonlinearity is derived by expanding the Sagdeev potential. Asymptotic solutions for different orders of nonlinearity are discussed for solitary waves. The present work is applicable to understanding the wave phenomena and associated nonlinear electrostatic perturbations in pair/bi-ion plasmas which may occur in space and laboratory plasmas.
\end{abstract}

Keywords Electrostatic solitons · Unmagnetized plasma model $\cdot$ Solitary waves $\cdot$ Pair-ion plasma .

Traped electrons $\cdot$ Sagdeev potential $\cdot$ Ion plasma waves · Ions acoustic waves

\footnotetext{
A. Mushtaq

Department of Physics, Abdul Wali Khan University Mardan,

23200, Mardan, Pakistan
}

M. Ikram ( $₫)$

Department of Physics, Hazara University, 21300 Mansehra, Pakistan

e-mail: mikphysics@gmail.com

\section{R. E. H. Clark}

Departments of Physics and Astronomy,

University of Texas at San Antonio,

San Antonio, USA

\section{Introduction}

In the typical geometric arrangement, 60 carbon atoms form a fullerene molecule. Recently, Oohara and collaborators generated a very dense pair-ion (PI) fullerene $C_{60}^{ \pm}$ plasma consisting of ions having equal masses and opposite charges $[1,2]$. They reported the existence of three kinds of waves, namely, ion plasma waves (IPW), ion acoustic (IA) waves, and intermediate frequency waves (IFW) (propagating parallel to the ambient magnetic field). Some of the detailed measurements relevant to the IA mode were presented in $[3,4]$. These measurements reveal that the IA wave actually has two separate branches, accompanied by some extra backward propagating mode situated between them. The IF mode has been the focus of a number of works, as the behavior of this mode does not fit into the standard plasma theory [5]. In the view of some authors [5], the IA wave also seems to be problematic as it should not appear in the perfectly antisymmetric PI plasma; however, an electrostatic acoustic mode may appear, if the PI plasma is non-isothermal $\left(T_{+} \neq T_{-}\right.$, where $T_{+}$and $T_{-}$are the temperatures of positive and negative ions). It is also theoretically argued that the existence of the IA mode and the increased values of the IA speed in Fig. 2 of [2] are the direct result of the existence of electrons in the PI fullerene plasma. PI plasmas also imply the existence of a PI hydrogen plasma as Ref [3], where the concept of a hydrogen PI plasma $\left(\mathrm{H}^{+}, \mathrm{H}^{-}\right)$with experimental configuration is proposed and presented. Since it is not very clear that pure PI plasmas are really produced in laboratory experiments, we thus consider a PI plasma with some fraction of electrons. The pair ion plasma is both accessible and sufficiently longlived that the collective behavior peculiar to the plasma state can be experimentally investigated under controlled conditions. This feature of the PI plasma attracted the attention 


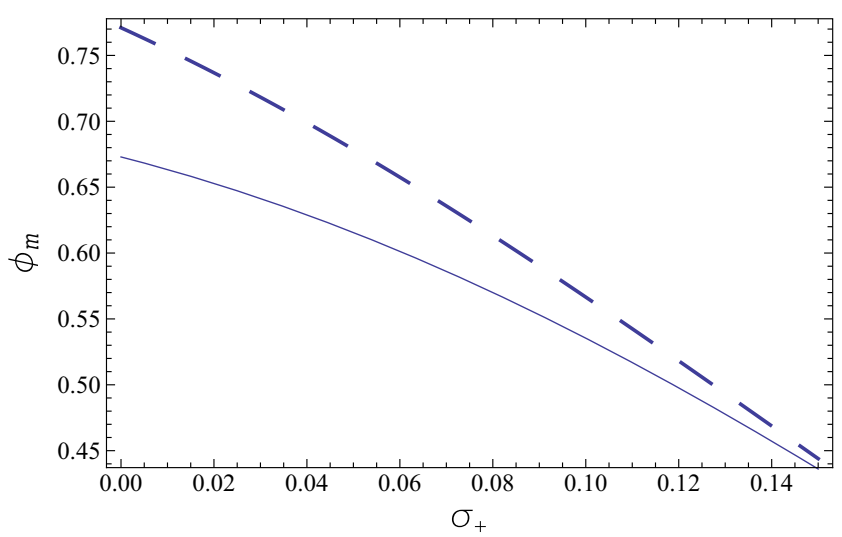

Fig. 1 (color online) Variation of the maximum amplitude $\phi_{m}$ of (21) against the positive ion temperature $\sigma_{+}$for different values of negative ion temperature $\sigma_{-}=0.25$ (solid curve) and $\sigma_{-}=0.2$ (dashed curve). Other parameters are $M=1.2, \beta=0.5$, and $\mu=0.5$

of researchers and series of papers appeared in the literature [6-12]. The PI plasma provides an alternate and comfortable way to study collective modes as compared to electronpositron (ep) plasma believed to have existed in the early universe, and in many astrophysical environments such as in the magnetosphere of pulsars, in active galactic nuclei, and also in some laboratory applications. Therefore, the study of a PI plasma can be regarded as an indirect way to understand the behavior of the ep plasma.

Besides the PI plasmas at the laboratory level, bi-ion plasmas are found in space plasmas. The bi-ion plasmas with different composition systems like $\mathrm{Ar}^{+}$plasmas with $\mathrm{F}^{-}$ions, $\mathrm{H}^{+}$plasmas with $\mathrm{O}_{2}^{-}$ions, $\mathrm{H}^{+}$plasmas with $H^{-}$ions, and so on occur in the D-region of the ionosphere [13] and also in the Martian magnetosphere [14]. The existence of a second ion population leads to an additional coupling between the ions and electrons through the Lorentz force (in magnetized plasmas) and charge neutrality. Even a small component of heavy ions in a proton-electron plasma may substantially alter the plasma wave and flow properties. The multi-ion nature of space plasmas gives rise to interesting effects and supports strong low frequency (and hence typically long wavelength) electrostatic perturbations in the form of ion acoustic waves. When the amplitudes of the waves are large, nonlinear effects, in particular ion acoustic solitons, become important in their propagation. Recently, Kim and Merlino [15] studied experimentally the formation of negative ions in a thermally ionized potassium plasma and observed the electrostatic ion cyclotron wave spectrum in a plasma containing $\mathrm{K}^{+}$positive ions (39 amu), electrons, and $\mathrm{C}_{7} \mathrm{~F}_{14}^{-}$(350 amu) negative ions. The bi-ion plasmas have applications in the earth's ionosphere, mesosphere, solar atmosphere, and in micro-electronic plasma processing reactors.
Usually, in an IA soliton, ions are inertial and electrons are Maxwellian. However, in practice, the electrons will not have a Maxwellian distribution due to the formation of phase space holes caused by the trapping of electrons in the IA wave potential. Thus, in most space plasmas, the electrons follow a trapped particle distribution when the particles obey the Maxwellian distribution at equilibrium [16, 17]. Very recently, some authors[18, 19] have studied the complex plasma dynamics by using the vortex-like electron distribution deriving the nonlinear evolution equations by using either a pseudo potential approach or the reductive perturbation technique. Some authors [20] have studied the ion-acoustic solitons in a plasma composed of warm positive and negative ions with different concentration of masses, charged states, and nonisothermal electrons, by using quasipotential method. Dust ion acoustic solitons in an unmagnetized pair-ion plasmas with adiabatic pair-ions, non-isothermal electrons, and negatively charged background dust were studied by Mushtaq et al. [21] by using both the small and arbitrary amplitude techniques. They have derived the integral equation involving the Sagdeev potential and Schamel-Korteweg-de Vries $(\mathrm{S}-\mathrm{KdV})$ equation with mixed-nonlinearity. The authors are discussing the applicability of work in the doped pair ion plasmas, not completely filtered and enriched with an extra massive charged component (e.g., dust defects). We in this work used the same method as adopted by $[20,21]$ in the PI plasmas in the presence of non-isothermal electrons but for specific subsonic adiabatic density compression. Also, we explain the large amplitude soliton in detail with mixed nonlinearity due to resonant electron contribution in the absence of any dust defects.

Ion-acoustic solitons in a plasma with negative ions have been observed experimentally by Ludwig et al. [22], where rarefaction solitons are produced by a filament-anode $50-\mathrm{V}$ $\mathrm{dc}$ discharge in a mixture of argon and sulfur hexafluoride. In this experimental setup, four ion-species plasma composed of positive $\mathrm{Ar}^{+}$ions, positive $\mathrm{SF}_{5}^{+}$ions, negative $\mathrm{F}^{-}$ions, negative $\left(\mathrm{SF}_{5}^{-}\right)$ions, and electrons are produced by the introduction of a gas with a large electron-attachment cross section, such as $\mathrm{SF}_{6}$ into the discharge chamber. Rarefactive solitons have been observed in this multicomponent plasma with negative ions when the negative-ion concentration is larger than some critical value. The observation of fast ion-acoustic rarefaction solitons are compared with theoretically derived $\mathrm{KdV}$ equation for this sort of plasma. Another experimental observation of ion-acoustic solitons in a multicomponent plasma with negative ions has been reported by Nakamura and Tsukabayashi [23]. At some critical concentration of negative ions, they have observed both the compressive and rarefactive solitons.

Thus, in the present investigation, we consider nonlinear propagation of sound waves in unmagnetized PI plasmas in 
the presence of non-isothermal electrons and with ions adiabaticity. We use the quasi-potential approach to study the characteristics of IA solitons in such a plasma. In Section 2, basic equations of plasma dynamics are used to derive an energy integral equation with the Sagdeev potential. Section 3 is devoted to a limited small amplitude soliton with the derivation of the modified Korteweg-de Vries $(\mathrm{KdV})$ equation. Finally, in Section 4, the conclusions of the present work are presented.

\section{Basic Equations and Localized Waves}

The unmagnetized plasma model is made up of pair ions (PI), i.e., positive ions (label +), negative ions (label -), and non-isothermal electrons (label $e$ ). We consider a fluid adiabatic PI of temperature $T_{ \pm}$and density $n_{ \pm}$. At equilibrium, the charge neutrality condition requires that $\sum_{j} q_{j} n_{j 0}=0$, with $q_{j}$ being the charge of the $j$ th species i.e., $q_{j}$ equals $-e$ for electrons, $e Z_{+}$for positive ions, and $-e Z_{-}$for negative ions where $Z_{+}$and $Z_{-}$are the charging state of the positive, negative ions, and $n_{j 0}$ is the equilibrium particle number density. The pair ions are assumed to be adiabatic and are described by standard fluid equations. These are the normalized continuity equations

$\frac{\partial n_{ \pm}}{\partial t}+\frac{\partial\left(n_{ \pm} v_{ \pm}\right)}{\partial x}=0$

the equations of motion,

$\frac{\partial v_{ \pm}}{\partial t}+v_{ \pm} \frac{\partial v_{ \pm}}{\partial x}+\frac{\sigma_{ \pm}}{n_{ \pm}} \frac{\partial p_{ \pm}}{\partial x} \pm Z_{ \pm} \frac{\partial \phi}{\partial x}=0$

and the adiabatic pressure equations,

$\frac{\partial p_{ \pm}}{\partial t}+v_{ \pm} \frac{\partial p_{ \pm}}{\partial x}+3 p_{ \pm} \frac{\partial v_{ \pm}}{\partial x}=0$

For adiabatic PI pressure, we have, $p_{ \pm}=p_{ \pm 0}\left(\frac{n_{ \pm}}{n_{ \pm 0}}\right)^{\gamma}$, where $\gamma=\frac{N+2}{N}$ is the polytropic index, $N$ being the number of degrees of freedom, and $p_{ \pm 0}=n_{ \pm 0} K_{B} T_{ \pm}$is the equilibrium PI pressure. Here, $n_{ \pm}, v_{ \pm}$, and $p_{ \pm}$are the normalized densities, fluid velocities and pressures of the positive and negative ions, respectively, and $\sigma_{ \pm}=\frac{T_{e}}{T_{ \pm}}$. The independent variables, $x$ and $t$, are normalized to a mixed electron-positive ion Debye length $\lambda_{D}=\sqrt{\frac{T_{e}}{4 \pi n_{+0} e^{2}}}$ and the inverse positive ion plasma frequency $\omega_{p+}=$ $\sqrt{\frac{4 \pi n_{+0} e^{2}}{m_{+i}}}$, respectively. The dependent variables $n_{ \pm}, v_{ \pm}$, and $p_{ \pm}$are normalized by the density $n_{ \pm 0}$, the ion acoustic speed $c_{s}=\sqrt{K_{B} T e / m_{+i}}$, and the equilibrium pressure $p_{ \pm 0}$, respectively. The electrostatic potential $\phi$ is normalized by $\frac{T_{e}}{e}$.

In addition to the PI species, we consider non-isothermal electrons. The model of non-isothermality is based on the assumption that some electrons could interact with the wave potential during its evolution and therefore can be trapped in the wave potential. To model an electron distribution with trapped particles, we employ the vortex-like electron distribution function [24, 25], which is an exact solutions of the electron Vlasov equation. Thus, the electron number density in the presence of trapped electrons can be expressed as

$n_{e}=e^{\phi} \operatorname{erf} c(\sqrt{\phi})+\frac{e^{\beta \phi}}{\sqrt{\beta}} \operatorname{erf}(\sqrt{\beta \phi}) \quad(\beta \geq 0)$

where $\beta=\frac{T_{e}}{T_{t}}$ is the ratio of free electron to trapped electron temperature. The complementary error function is defined as $\operatorname{erf} c(\phi)=1-\operatorname{erf}(\phi)$ where $\operatorname{erf}(\phi)=\frac{2}{\sqrt{\pi}} \int_{0}^{\phi} e^{-t} d t$ is the error function. Finally, the set of equations is closed by Poisson's equation

$\frac{\partial^{2} \phi}{\partial x^{2}}+Z_{+} n_{+}-\mu n_{e}-Z_{-}(1-\mu) n_{-}=0$,

with $\mu=\frac{n_{e 0}}{n_{+0}}$. For the localized stationary solution, we define a transformed coordinate $\xi$ such that, $\xi=x-M t$, where $M=u / c_{s}$ is the Mach number and $u$ is the speed of the localized nonlinear structure moving with the frame. Integrating (1) and (2) with boundary conditions $n_{ \pm} \rightarrow 1$, $v_{ \pm} \rightarrow 0$, and $\phi \rightarrow 0$ as $\xi \rightarrow \pm \infty$, we obtain a general solution for PI in terms of $n_{ \pm}^{2}$ as

$n_{ \pm}^{2}=\frac{1}{6 \sigma_{ \pm}}\left[\chi_{ \pm} \pm \sqrt{\chi_{ \pm}^{2}-12 \sigma_{ \pm} M^{2}}\right]$

where $\chi_{ \pm}=M^{2}+3 \sigma_{ \pm} \mp 2 Z_{ \pm} \phi$. The + sign (inside the equation) has to be used for a subsonic species $(M<1)$ and the - sign for a supersonic one $(M>1)$. Following the technique of [26-28], we look for solutions for $n_{ \pm}$in the form

$n_{ \pm}=\frac{1}{\sqrt{2}}[\sqrt{X} \pm \sqrt{Y}]$

Using (6) in (5) will allow us to determine $X$ and $Y$ as

$$
\begin{aligned}
& X=\frac{1}{6 \sigma_{ \pm}}\left[\left(M+\sqrt{3 \sigma_{ \pm}}\right)^{2} \mp 2 Z_{ \pm} \phi\right] \\
& Y=\frac{1}{6 \sigma_{ \pm}}\left[\left(M-\sqrt{3 \sigma_{ \pm}}\right)^{2} \mp 2 Z_{ \pm} \phi\right]
\end{aligned}
$$


Using (8) and (9), some standard algebraic manipulation leads to the following expressions for $n_{+}$and $n_{-}$

$$
\begin{aligned}
n_{+}=\frac{1}{2 \sqrt{3 \sigma_{+}}} & {\left[\left\{\left(M+\sqrt{3 \sigma_{+}}\right)^{2}-2 Z_{+} \phi\right\}^{\frac{1}{2}}\right.} \\
\pm & \left.\left\{\left(M-\sqrt{3 \sigma_{+}}\right)^{2}-2 Z_{+} \phi\right\}^{\frac{1}{2}}\right] \\
n_{-}=\frac{1}{2 \sqrt{3 \sigma_{-}}}\left[\left\{\left(M+\sqrt{3 \sigma_{-}}\right)^{2}+2 Z_{-} \phi\right\}^{\frac{1}{2}}\right. & {\left[\{\}^{\frac{1}{2}}\right] } \\
\pm & \left.\left\{\left(M-\sqrt{3 \sigma_{-}}\right)^{2}+2 Z_{-} \phi\right\}^{2}\right]
\end{aligned}
$$

Again, the \pm signs refer to subsonic/supersonic species. Using all the constituent densities from (4), (10), and (11) into (5), a multiplication by $\frac{d \phi}{d \xi}$ and an integration give us the usual energy integral equation as

$\frac{1}{2}\left(\frac{d \phi}{d \xi}\right)^{2}+V(\phi)=0$,

where $V(\phi)$ is the pseudo-potential (or Sagdeev potential) which can be expressed as

$$
\begin{array}{r}
V(\phi)=\mu\left[1-P_{e}(\phi, \beta)\right]+\frac{1}{6 \sqrt{3 \sigma_{+}}} \\
{\left[\left(M+\sqrt{3 \sigma_{+}}\right)^{3} \pm\left(M-\sqrt{3 \sigma_{+}}\right)^{3}\right]} \\
-\frac{1}{6 \sqrt{3 \sigma_{+}}}\left\{\left[\left(M+\sqrt{3 \sigma_{+}}\right)^{2}-2 Z_{+} \phi\right]^{\frac{3}{2}}\right. \\
\pm \frac{(1-\mu)}{6 \sqrt{3 \sigma_{-}}\left[\left(M+\sqrt{3 \sigma_{-}}\right)^{3} \pm\left(M-\sqrt{3 \sigma_{-}}\right)^{3}\right]} \\
-\frac{(1-\mu)}{6 \sqrt{3 \sigma_{-}}}\left\{\left[\left(M+\sqrt{3 \sigma_{+}}\right)^{2}-2 Z_{+} \phi\right]^{\frac{3}{2}}\right\} \\
\left. \pm\left[\left(M-\sqrt{3 \sigma_{-}}\right)^{2}+2 Z_{-} \phi\right]^{\frac{3}{2}}\right\}
\end{array}
$$

here the function $P_{e}(\phi, \beta)$ are given by [24, 25]

$$
\begin{array}{r}
P_{e}(\phi, \beta)=\exp (\phi)[1-\operatorname{erf}(\sqrt{\phi})]+\frac{2 \sqrt{\phi}}{\sqrt{\pi}}\left(1-\frac{1}{\beta}\right) \\
+\frac{\exp (\beta \phi)}{\beta \sqrt{|\beta|}} \operatorname{erf}(\sqrt{\beta \phi}),
\end{array}
$$

and shows the electron and ion pressures at $\phi$. To obtain (12), we have used the boundary conditions that as $\xi \rightarrow$ $\pm \infty, \phi \rightarrow 0$ and $\frac{d \phi}{d \xi} \rightarrow 0, \operatorname{erf}(\sqrt{\beta \phi}) \rightarrow 0$, and $e^{\phi} \operatorname{erf} c(\sqrt{\phi}) \rightarrow 1$. The relation (12) is a well known equation in the form of the 'energy integral' of an oscillating particle of unit mass in a conservative force field with velocity $\frac{d \phi}{d \xi}$, position $\phi$ and pseudo-time $\xi$ in a potential well $V(\phi)$. The conditions for the existence of a localized solution of (12) require that (i) $V(0)=\left.\frac{\partial V(\phi)}{\partial \phi}\right|_{\phi=0}=0$ and (ii) $\left.\frac{\partial^{2} V(\phi)}{\partial \phi^{2}}\right|_{\phi=0}<0$ (where the fixed point at $\phi \rightarrow 0$ is unstable). Condition (i) is satisfied by (13). Besides that $V(\phi)$ should be negative between $\phi=0$ and $\phi_{m}$, where $\phi_{m}$ is the maximum (or minimum) value of $\phi$ for which $V\left(\phi_{m}\right)=0$. The second condition, which is often referred to as the soliton condition and can be expressed (for the supersonic species) as

$\left.\frac{\partial^{2} V(\phi)}{\partial \phi^{2}}\right|_{\phi=0}=-\mu+\frac{1}{M^{2}+3 \sigma_{+}}+\frac{(1-\mu)}{M^{2}+3 \sigma_{-}}<0$

(14) can be treated as a quadratic equation in $M^{2}$ by considering $M=M_{s}$ at $\left.\frac{\partial^{2} V(\phi)}{\partial \phi^{2}}\right|_{\phi=0}=0$, so that

$M^{2}>M_{s}^{2}=\frac{b}{2 a}\left[1+\left(1-\frac{4 a c}{b}\right)^{\frac{1}{2}}\right]$

where $a=\mu, b=Z_{+}+(1-\mu) Z_{-}+3 \mu\left(\sigma_{+}+\sigma_{-}\right)$, and $c=3(1-\mu) \sigma_{+} Z_{-}+9 \mu \sigma_{+} \sigma_{-}+3 \sigma_{-} Z_{+}$. Reference (15) gives the lower limit for $M$ in the presence of non-isothermal electrons, which correspond to the true ionacoustic velocity in the given plasma model. In the absence of negative ions $(\mu=1)$, and assuming positive ions are cold $\left(\sigma_{+}=0\right)$, with $Z_{ \pm}=1$,we obtain $M_{s}=1$ (namely, the speed of ion-acoustic waves in electron-ion plasmas). For cold negative $\left(\sigma_{-}=0\right)$ and adiabatic positive ions with $Z_{ \pm}=1$, an expression for $M_{S}$ is

$M_{s}^{2}=\left(2-\mu+3 \sigma_{+} \mu\right)\left[1+\sqrt{1-\frac{2 \sigma_{+}(1-\mu) \mu}{\left(2-\mu+3 \sigma_{+} \mu\right)^{2}}}\right]$,

similarly, for cold pair ions $\left(\sigma_{+}=\sigma_{-}=0\right)$, we have $M_{s}=\sqrt{2(2-\mu)}$. For bounded solution, we have checked that $\phi\left(x_{\min }\right)=0,\left.\Longrightarrow V(\phi)\right|_{\phi\left(x_{\min }\right)}=0$. Now, we find out the second condition of the soliton solution and that gives us

$\left.V(\phi)\right|_{\phi\left(x_{\max }\right)}=0$, where $\phi\left(x_{\max }\right)=\phi_{m}$,

which yields a relation between the amplitude and velocity of the wave (Mach number) and can be regarded as the nonlinear dispersion relation (NDR) [24, 25], namely $V\left(\phi_{m}\right.$, $M, ..)=0$. Under this condition the NDR for supersonic 
species (using (13)) can be written as

$$
\begin{array}{r}
0=\mu\left[1-P_{e}\left(\phi_{m}, \beta\right)\right] \\
+(1-\mu)\left(M^{2}+\sigma_{-}\right)+M^{2}+\sigma_{+} \\
-\frac{1}{6 \sqrt{3 \sigma_{+}}}\left\{\left[\left(M+\sqrt{3 \sigma_{+}}\right)^{2}-2 \phi_{m}\right]^{\frac{3}{2}}\right. \\
\left.-\left[\left(M-\sqrt{3 \sigma_{+}}\right)^{2}-2 \phi_{m}\right]^{\frac{3}{2}}\right\} \\
-\frac{(1-\mu)}{6 \sqrt{3 \sigma_{-}}}\left\{\left[\left(M+\sqrt{3 \sigma_{-}}\right)^{2}+2 \phi_{m}\right]^{\frac{3}{2}}\right. \\
\left.-\left[\left(M-\sqrt{3 \sigma_{-}}\right)^{2}+2 \phi_{m}\right]^{\frac{3}{2}}\right\} .
\end{array}
$$

(17) provides the upper limit of Mach number $M\left(\phi_{m}\right)$, which turns out to depend on the parameters $\mu, \sigma_{+}, \sigma_{-}$, and $\beta$. It is clear that for an arbitrary value of the amplitude $\phi_{m}$, a simple analytical relation between $\phi_{m}$ and $M$ for different values of $\mu, \sigma_{+}, \sigma_{-}$, and $\beta$ is not easy to obtain. Therefore, this relation can be solved numerically to find out that how a solitary wave speed $M$ changes with the above-mentioned parameters. From the requirement that the element of the square root is positive and real in (17), the domain of $\phi_{m}$ is determined to be confined in the regions $\phi_{m} \leq \phi_{c 1}$ and $\phi_{m} \leq \phi_{c 2}$, where $\phi_{c 1}=\frac{\left(M+\sqrt{3 \sigma_{+}}\right)^{2}}{2}$ and $\phi_{c 2}=\frac{\left(M-\sqrt{3 \sigma_{+}}\right)^{2}}{2}$. For cold positive and negative ions, the nonlinear dispersion relation (17), for given $\phi_{m}$ yields a relation as

$M^{2}=\frac{-\Theta^{2}}{2(1-\mu)\left[\Theta+\phi_{m}\right]}$,

where $\Theta=\mu\left[1-P_{e}\left(\phi_{m}, \beta\right)\right]$ with $P_{e}\left(\phi_{m}, \beta\right)$ is the electron pressure at $\phi_{m}$, affected by the number of trapped electrons. The relation (18) shows that the Mach number of solitary wave depends on different parameters of the medium.

\section{Small Amplitude Limit}

In order to confirm the possibility of localized ion-acoustic solitons in the pair ions-electron plasma, we also investigate the pseudo potential $V(\phi)$ in the small amplitude limit $(\phi<<1)$. Using Taylor's expansion, the Sagdeev potential $V(\phi)$ near $\phi=0$ can be expanded in powers of $\phi$ and, keeping up to the third-order terms, (13) then takes the form

$$
\left(\frac{d \phi}{d \xi}\right)^{2}+A \phi^{2}-B \phi^{5 / 2}-C \phi^{3}=0
$$

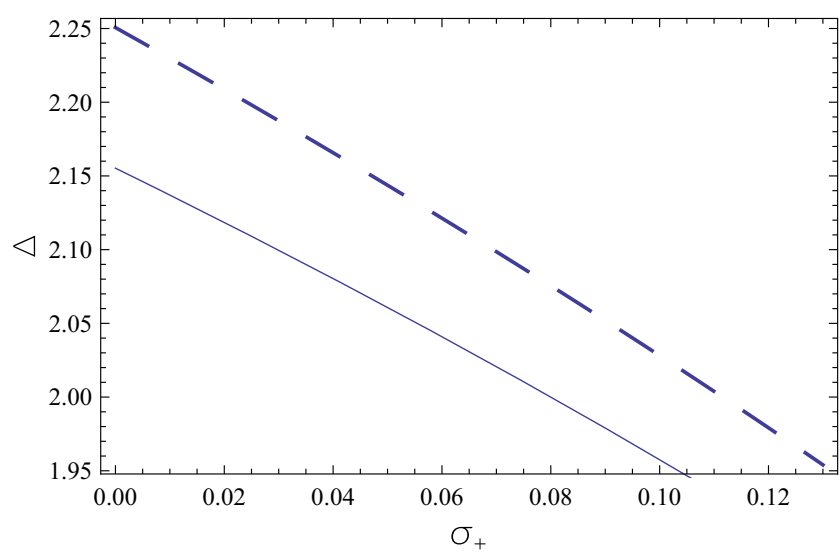

Fig. 2 (color online) Behavior of the width $\Delta$ of soliton (of (21)) against the positive ion temperature $\sigma_{+}$for different values of negative ion temperature $\sigma_{-}=0.23$ (solid curve) and $\sigma_{-}=0.2$ (dashed curve). Other parameters are the same as in Fig. 1

where

$$
\begin{aligned}
& A=\frac{1}{M^{2}-3 \sigma_{+}}+\frac{1-\mu}{M^{2}-3 \sigma_{-}}-\mu \\
& B \quad=\frac{2}{\beta \sqrt{\pi}}\left(1-\frac{6 \beta}{5}+\frac{\beta^{2}}{5}\right) \mu \\
& C=\frac{\mu}{3}-\frac{M^{2}+3 \sigma_{+}}{\left(M^{2}-3 \sigma_{+}\right)^{3}}-\frac{\left(M^{2}+3 \sigma_{-}\right)(1-\mu)}{\left(M^{2}-3 \sigma_{+}\right)^{3}}
\end{aligned}
$$

After the integration of general (19) with respect to $\xi$, along with the allowable boundary conditions, we get the following general soliton solution

$\phi=\left[\frac{B}{2 A}+\left(\frac{B^{2}}{4 A^{2}}+\frac{C}{A}\right)^{1 / 2} \cos h\left(\frac{\xi}{\Delta}\right)\right]^{-2}$

Here, $\Delta=(4 / A)^{1 / 2}$ gives the width of solitary waves. Solution (21) is the same as that of the exact solution of the generalized $\mathrm{KdV}$ equation of [29] with different orders of nonlinearity. It shows that the amplitude $\phi_{m}=\phi(\xi=0)$ depends on the trapped electron distribution, density concentration, and adiabaticity of PI ions during the formation of solitary waves. Variations of the small amplitude $\phi_{m}$ and the width $\Delta$ as a function of positive ion temperature $\sigma_{+}$for different values of negative ion temperature $\sigma_{-}$have been depicted in Figs. 1 and 2. It can be seen from these figures that both amplitude and width decrease with the PI temperatures. This shows that adiabatic PI conditions tend to oppose the formation of localized electrostatic structure.

We will now focus our attention on two asymptotic cases of solution (21), which give some insights to the contribution of resonant electrons. First, we consider a case where we assume that $B>C$, meaning that the coefficients $B$ and $C$ of the nonlinear terms in (19) are such that the 
term with $B$ dominates. The solution (21), after expansion in powers of $1 / B$ takes the form

$$
\begin{array}{r}
\phi=\left(\frac{A}{B}\right)^{2}\left[1-\frac{2 A C}{B^{2}} \cosh \left(\frac{\xi}{\Delta}\right) \operatorname{sech}\left(\frac{\xi}{2 \Delta}\right)\right] \\
\sec h^{4}\left(\frac{\xi}{2 \Delta}\right)
\end{array}
$$

The number of resonant electrons and the adiabatic temperature of the ions affect the amplitude of IA solitary waves. If the effect of nonlinear resonant interaction of electrons due to trapping becomes so dominant that $C$ can be neglected in comparison to $B$, then (22) reduces to

$\phi=\frac{B}{A} \sec h^{4}\left(\frac{\xi}{2 \Delta}\right)$

On the contrary, if we suppose that $C>>B$, then the expansion of solution (21) in powers of $1 / C$ gives

$$
\begin{aligned}
\phi=\left(\frac{A}{C}\right) \sec h^{2} & \left(\frac{\xi}{\Delta}\right)\left[1-\frac{B}{\sqrt{A C}} \sec h\left(\frac{\xi}{\Delta}\right)\right. \\
& \left.-\frac{B^{2}}{4 A C}\left\{1-3 \sec h^{2}\left(\frac{\xi}{\Delta}\right)\right\}\right]
\end{aligned}
$$

In this case, if the effect of nonlinear resonant interaction due to electron trapping becomes negligible such that we ignore $B$ in comparison to $C$, then (24) reduces to

$\phi=\left(\frac{A}{C}\right) \sec h^{2}\left(\frac{\xi}{\Delta}\right)$

To examine the existence of regions in parametric space and the nature of the IA solitary excitation in the PI plasma, the numerical solutions of (12) and (13) have been analyzed using typical plasma parameters. In laboratory plasmas such as fullerene PI plasmas [1] or hydrogen PI plasmas [3] in the presence of electrons and background dust, we may choose $n_{ \pm} \sim\left(10^{7}-10^{10}\right) \mathrm{cm}^{-3}, T_{ \pm} \sim(1.5-2) \mathrm{eV}$, and $T_{e} \sim 35 \mathrm{eV}$ which give us finite values of the parameters used for numerical plotting. Since we are using the specific subsonic adiabatic density compression of equations (10) and (11), so we are getting the compression soliton structure as previously discussed in [21].

The effects of the Mach number $(M)$, electron density concentration $(\mu)$, and the nonlinear resonant effects due to electron trapping on the behavior of electrostatic solitary wave are displayed in Figs. 3-5. These results show that our present plasma model permits only compressive solitons. The Sagdeev potential curves and the corresponding solitary wave profiles for different values of $M$ are plotted in Fig. 3 . It can be seen from the upper panel of Fig. 3 that as we increase the values of $M$, the depth of the Sagdeev potential increases on the positive $\phi$-axis, this is a consequence of the compressive solitary waves with larger amplitude (lower panel of Fig. 3).
A Sagdeev potential (13) as a function of electrostatic potential $\phi$ for different values of electron density concentration $\mu$ is shown in the upper panel of Fig. 4, and the corresponding soliton profiles for different values of $\mu$ are shown in the lower panel of Fig. 4. For higher values of $\mu$, the amplitude of solitary structure increases while the width is decreasing. The increasing $\mu$ gives the increasing electron thermal energy. In order to conserve the energy of the system, the electron thermal energy should be decreased and the ion energy should be increased. The increased ion energy will increase the height and amplitude of solitons in PI plasmas. This sort of trend is the same for dust ion acoustic waves, as observed in [21](Fig. 4 therein).

The impact of the resonant particle effects due to trapping of electrons on the Sagdeev potential and solitary wave is displayed in Fig. 5, where the larger values of $\beta$ reduce the amplitude of the Sagdeev potential having wider solitary structures with shallower density and electric field depletions. This means that the amplitude decreases
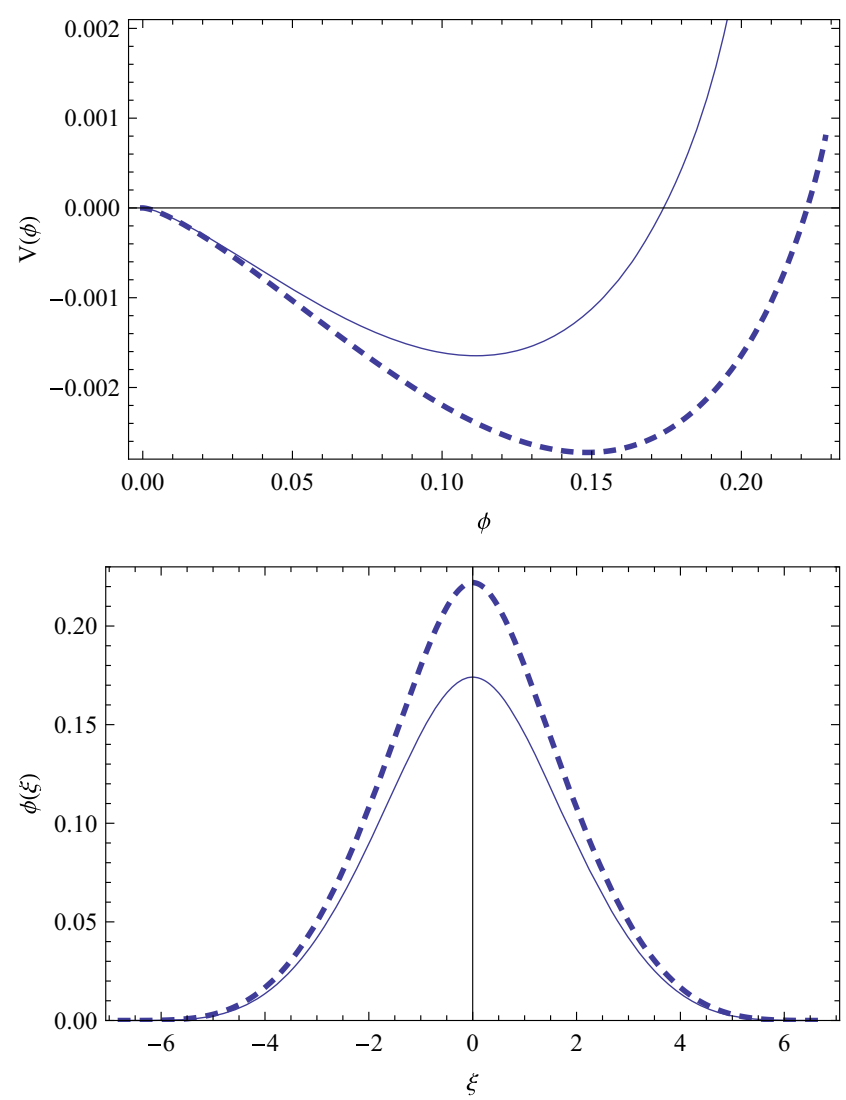

Fig. 3 (color online) Behavior of the Sagdeev potential $V(\phi)$ (upper panel) and the corresponding solitary wave profiles $\phi(\xi)$ (lower panel) for different values of Mach number $M=1.4$ (solid curve) and $M=1.45$ (curve). Other parameters are $\beta=0.8, \mu=0.5 \sigma_{+}=0.2$, and $\sigma_{-}=0.1$ 

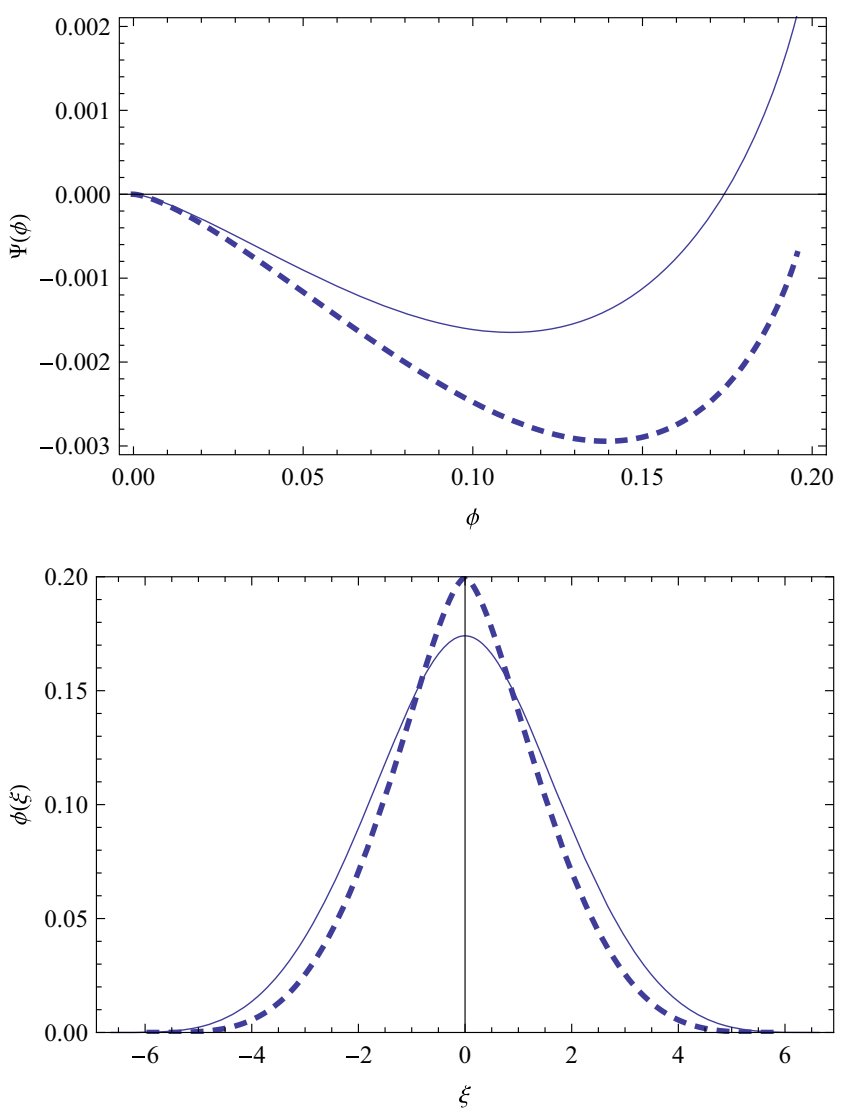

Fig. 4 (color online) Pseudopotential $V(\phi)$ as a function of electrostatic potential $\phi$ (upper panel) and the corresponding solitary wave profiles $\phi(\xi)$ (lower panel) for different values of electron density concentration $\mu=0.5$ (solid curve) and 0.55 (dashed curve) for fixed value of $M=1.4$. Other parameters are the same as in Fig. 3

with the increased values of $\beta$ and shows that the nature of the trapping effect is a sort of mordant for the formation of electrostatic solitary structure. This is the same trend as illustrated in [21] for dust ion acoustic waves (Fig. 3 therein).

\section{Summary and Conclusion}

We have studied the effects of PI temperature adiabaticity, particle density concentration, and nonlinear resonant particle effects, such as trapping of electrons, on the arbitrary amplitude of IA solitons in PI-electron plasmas. The electrons are assumed to follow a vortex-like distribution. The solitary wave exists because of the counterbalance between the nonlinearity induced by the electron trapping and ion fluid convection and the dispersion due to temperature effects. Using the pseudopotential approach, we have derived an energy integral equation involving the Sagdeev potential. In the case of negative ions, we have shown
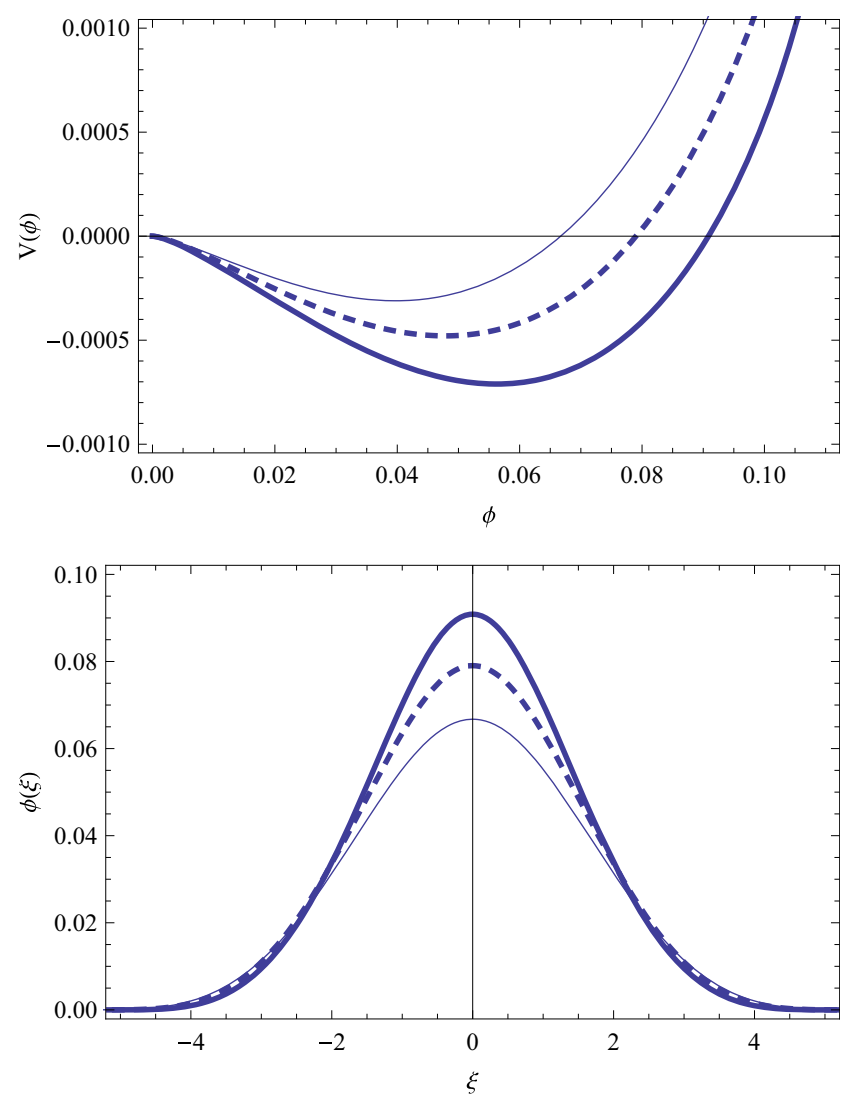

Fig. 5 (color online) Sagdeev potential $V(\phi)$ (upper panel) and the corresponding solitary wave profiles $\phi(\xi)$ (lower panel) for different values of the resonant electron parameter. $\beta=0.8$ (solid curve), 0.78 (dotted curve), and 0.76 (bold curve) for fixed value of $M=1.4$. Other parameters are the same as in Fig. 3

that the model supports both positive and negative potential solitons, where the Mach number for positive (negative) potential solitons is limited from above by the condition at which the positive ion density becomes complex. From the energy integral equation, a generalized $\mathrm{KdV}$ equation with mixed orders of nonlinearity has been derived (by expanding the Sagdeev potential) along with its general solution. The effects of ion adiabaticity on small amplitude and width have been discussed. Solutions involving lower and higher nonlinear orders have also been deduced from the general solution. The above mentioned effects are found to change the profile of solitary waves quite significantly. The electron concentration gives a strong and enhancing effect on the existence domain of positive solitons while the PI temperatures have effects which weaken the soliton amplitude. It is found that the characteristics of nonlinear IA waves vary substantially due to the nonisothermality of electrons. The amplitude and width of the solitons are different when some electrons are non-isothermal from the case when they all are isothermal. This means that the transformation from 
non-isothermal to isothermal phase is nonuniform. It is also found that the amplitude of solitons in the case of isothermal electrons is larger than that for trapped electron distributions, which implies that trapped electrons induced a kind of inertia in the propagation of nonlinear electrostatic perturbation. Our this analytical work, i.e., propagation of ion acoustic soliton in pair-ion plasmas in the presence of nonisothermal electrons are supported by some experimental observations as investigated in [23], where both compressive and rarefactive solitons are observed under certain critical condition. Since we are using the subsonic adiabatic density compression of positive and negative ions, so under this specific condition we are getting the compression soliton structure only. Also, it is concluded that ion-acoustic waves are likely to be found in non-isothermal pair-ions plasmas in the same way as solitons are existed in the non-isothermal electron-positron plasmas [30-32]. From our numerical results, we also observed that temperature effects due to both type of ions are destructive and reduce the speed of solitons. These temperature effects are consistent with the results of [31,32], where the destructive nature of Alfven solitons (due to temperature) are observed in pair plasmas with finite temperatures. Our present results can be useful in understanding wave phenomena and associated nonlinear electrostatic structures in PI/bi-ion plasmas which may occur in space and at laboratory level.

\section{References}

1. W. Oohara, R. Hatakeyama, Phys. Rev. Lett. 91, 205005 (2003)

2. W. Oohara, D. Date, R. Hatakeyama, Phys. Rev. Lett. 95, 175003 (2005)

3. W. Oohara, R. Hatakeyama, Phys. Plasmas 14, 055704 (2007)

4. W. Oohara, Y. Kuwabara, R. Hatakeyama, Phys. Rev. E. 75, 056403 (2007)
5. J. Vranjes, D. Petrovic, B.P. Pandey, S. Poedts, Phys. Plasmas 15, $072104(2008)$

6. S.K. El-Labany, W.M. Moslem, W.F. El-Taibany, M. Mahmoud, Phys. Scr. 70, 317 (2004)

7. I. Kourakis, A. Esfandyari-Kalejahi, M. Mehdipoor, P.K. Shukla, Phys. Plasmas 13, 052117 (2006)

8. I. Kourakis, F. Verheest, N.F. Cramer, Phys. Plasmas 14, 022306 (2007)

9. J. Vranjes, S. Poedts, Plasma Sour. Sci. Technol. 14, 485 (2007)

10. W.M. Moslem, P.K. Shukla, Phys. Plasmas 13, 122104 (2006)

11. B. Zhao, J. Zheng, Phys. Plasmas 14, 062106 (2007)

12. A. Mushtaq, R. Saeed, Q. Haque, Phys. Plasmas 16, 084501 (2009). ibid 18, 042305, 2011

13. K. Mishra, R.S. Chhabra, Phys. Plasmas 3, 4446 (1996)

14. K. Sauer, E. Dubinin, K. Baumgärte, V. Tarasov, Earth Planets and Space 50, 269 (1998)

15. S.H. Kim, R.L. Merlino, Phys. Rev. E 76, 035401(R) (2007)

16. J. Srinivas, P.K. Shukla, Astrophys. Space. Sci. 219, 143 (1994)

17. P.K. Shukla, R. Bharuthram, Phys. Rev. A 34, 4457 (1986)

18. H. Alinejad, S. Sobhanian, J. Mahmoodi, Phys, Plasmas 13, 012304 (2006)

19. H. Alinejad, Phys. Scr. 81, 015504 (2010)

20. T.S. Gill, H. Kaur, N.S. Saini, Phys. Plasmas 10, 3927 (2003)

21. A. Mushtaq, M.N. Khattak, Z. Ahmad, Qamar. Phys, A. Plasmas 19, 042304 (2012)

22. G.O. Ludwig, J.L. Ferreira, Y. Nakamura, Phys. Rev. Lett. 52, 275 (1983)

23. Y. Nakamura, I. Tsukabayashi, Phys. Rev. Lett. 52, 2356 (1984)

24. H. Schamel, J. Plasma Phys. 14, 905 (1972)

25. H. Schamel, J. Plasma Phys. 9, 377 (1973)

26. F. Verheest, M.A. Hellberg, I. Kourakis, Phys. Plasmas 15, 112309 (2008)

27. S.S. Ghosh, K.K. Ghosh, A.N.S. Iyengar, Phys. Plasmas 3, 3939 (1998)

28. T.K. Baluku, M.A. Hellberg, I. Kourakis, N.S. Saini, Phys. Plasmas 17, 053702 (2010)

29. S.G. Tagare, A. Chakrabarti, Phys. Fluids 17, 1331 (1974)

30. A.C.-L. Chian, C.F. Kennel, Astrophys. Space. Sci. 97, 9 (1983)

31. F.A. Asenjo, F.A. Borotto, A.C.-L. Chian, V. Munoz, Valdivia, J. A., E.L. Rempel, Phys. Rev. E 85, 046406 (2012)

32. R.A. Lopez, F.A. Asenjo, V. Munoz, A.C.-L. Chian, J.A. Valdivia, Phys. Rev. E 88, 023105 (2013) 